\title{
Predicting major mental illness: ethical and practical considerations
}

Stephen M. Lawrie, Sue Fletcher-Watson, Heather C. Whalley and Andrew M. McIntosh

\section{Summary}

An increasing body of genetic and imaging research shows that it is becoming possible to forecast the onset of major psychiatric disorders such as depression and schizophrenia before people become ill with ever improving accuracy. Practical issues such as the optimal combination of clinical and biological variables are being addressed, but the application of predictive algorithms to individuals or in routine clinical settings have yet to be tested. The development of predictive methods in mental health comes with substantial ethical questions, including whether people wish to know their level of risk, as well as individual and societal attitudes to the potential adverse effects of data sharing, early diagnosis and treatment, which so far have been largely ignored. Preliminary data suggests that at least some people think predictive research is valuable and would take part in such studies, and some would welcome knowing the results. Future initiatives should systematically assess opinions and attitudes in conjunction with scientific and technical advances.

\section{Declaration of interest}

In the past 3 years, S.M.L. has received personal fees from Otsuaka, Sunovion and Janssen, and research grant support from Janssen and Lundbeck. A.M.M. has received research support from the Sackler Trust, Eli Lilly and Janssen. S.M.L. is part of the PSYSCAN consortium

\section{Keywords}

Bipolar affective disorders; depressive disorders; ethics; imaging; psychotic disorders.

\section{Copyright and usage}

(C) The Royal College of Psychiatrists 2019. This is an Open Access article, distributed under the terms of the Creative Commons Attribution-NonCommercial-NoDerivatives licence (http:// creativecommons.org/licenses/by-nc-nd/4.0/), which permits non-commercial re-use, distribution, and reproduction in any medium, provided the original work is unaltered and is properly cited. The written permission of Cambridge University Press must be obtained for commercial re-use or in order to create a derivative work.
One of the major limitations in the practice of psychiatry, and one of the perennial causes of controversy about the nature and management of mental disorder, is the general lack of diagnostic tests. This has led to a research thrust towards identifying 'biomarkers' clinical, blood, imaging, cognitive or other measures that have the ability to accurately forecast the onset of illness in individuals without clinical disorder, or provide additional diagnostic or prognostic information in those presenting to clinical care. Arguably, most valuable of all would be a test that could predict treatment response or the lack of it. Some progress has been made in early and differential diagnostic approaches to dementia and the practice of old age psychiatry already includes routine (early) diagnostic work-ups. Some advances have been made in schizophrenia, with glimpses of potential in depression. ${ }^{1}$ But the practical and ethical considerations of making early diagnoses in general adult psychiatry have received at most brief consideration. ${ }^{2,3}$ This overview considers the main issues and makes suggestions for researchers in the field.

\section{Predictive testing - the state of the science}

A systematic review of risk prediction models in psychiatry up until 2013 identified 12 reported models for major depression and 24 for schizophrenia or other primary psychotic disorders, among 1 or 2 reports for other conditions. ${ }^{4}$ Almost all of these studies have been limited by small sample sizes and a lack of independent data test sets. Studies on depression have rarely included biological variables, although a few are now available. ${ }^{5}$

Most progress has been made in predicting broadly defined 'psychosis', usually defined as the presence of above-threshold delusions, hallucinations or formal thought disorder. Several models having suitably robust diagnostic parameters such as an 'area under the curve' (AUC) of around 0.8 - the level at which screening programmes tend to be considered for implementation. Note however that the prevalence of a condition is a critical consideration here, because as the prevalence falls, the false-positive rate inevitably rises. Accordingly, screening for psychosis in the general population is not desirable and most of these studies have examined people with subthreshold psychotic symptoms presenting to 'clinical high risk' research services. Although transition rates vary, the generally observed rate of transition to 'psychosis' is $30-40 \%$ over $2-3$ years of follow-up. Across studies, subthreshold symptom severity, poorer functioning and a family history of schizophrenia are consistent clinical predictors of transition. ${ }^{6,7}$ Biological approaches tend to have focused on neuroimaging indices, usually involving complex machine learning analyses, some of which have since been replicated and validated in independent samples, ${ }^{8}$ but these clinical and neuroimaging research streams have largely progressed in parallel. Indeed, the only study we are aware of which has systematically evaluated various combinations of clinical and biological parameters, and validated the predictive algorithm across multiple sites, reported no advantage of including imaging data. ${ }^{9}$

The recent and ongoing discovery of numerous genetic risk factors for schizophrenia and depression has opened up additional predictive possibilities that have yet to be incorporated into multivariate analyses. ${ }^{10}$ In schizophrenia, a genome-wide association study (GWAS) of more than 100000 case-participants and controls identified 108 loci associated with schizophrenia. Calculating a polygenic risk score (PRS) for schizophrenia based on whether individuals had the risk variant at each locus, multiplied by the odds ratio for that locus, captured about $7 \%$ of the variance in disease liability in independent populations. This translates into an odds ratio elevated $8-20 \times$ when comparing the highest and lowest decile groups, depending on population. ${ }^{11}$ It should be noted, however, that PRSs are not as yet validated in culturally diverse groups - although this is a priority for the Psychiatric Genomics 
Consortium (http://www.med.unc.edu/pgc/). Similarly, for depression, a recent GWAS in 135458 case-participants and 344901 controls identified 44 independent and significant loci, and those in the highest decile of PRS risk have an increased odds ratio of $2.5 \times$ of depression. ${ }^{12}$ Increasing the numbers in GWASs has already and will further increase the number of 'hits', the explained variance and predictive power. Moreover, a PRS seems to add predictive power to simply having a family history. ${ }^{13,14}$

Genetic and other biomarkers for diagnosis are not necessarily the same as those for treatment response. Symptom severity and early response are generally useful clinical guides but one hardly needs complex analyses to use them in clinical practice. ${ }^{15}$ Pharmacogenomic studies have thus far proved disappointing, although PRSs and epigenetic markers such as DNA methylation show some promise. ${ }^{16}$ There are other candidate biomarkers for treatment response in first-episode psychosis, ${ }^{17}$ but they have yet to be examined in multivariate studies. More progress has been made with predicting antidepressant response in depression through, for example, neuroimaging of anterior cingulate cortex activity $^{18}$ and in particular with machine learning models of multiple data types. ${ }^{19}$ Still greater predictive power may accrue by identifying neurophysiological subtypes that differentially predict response to different treatments. ${ }^{20}$

\section{Practical and ethical issues to be addressed}

Evidence-based medicine guidelines ${ }^{21}$ recommend four steps in establishing a validated predictive tool and decision rules for use in clinical practice: selecting variables, validation at a single site, validation at different sites and establishing the impact on clinical practice. We now have at least proof of concept for the first three steps in predicting 'psychosis'. Further work along those lines is, however, likely to be required for particular populations and needs to demonstrate that the prediction tool can change clinician behaviour and benefit patients. Several hurdles remain, however, to be negotiated, as follows.

\section{Optimising prediction across research centres}

There are already several large consortia (for example PSYSCAN: http://psyscan.eu and PRONIA http://www.pronia.eu) developing enhanced multivariate depression and psychosis prediction tools. Their general approach is to use machine learning to combine clinical, behavioural, cognitive, imaging and genetic data from different modalities to optimise predictive algorithms. These will be tested against what clinical trial and natural treatment history data are available. Harmonising and standardising clinical, behavioural and cognitive test administration and scoring are complex but tractable concerns. Varying approaches to clinical management and biological measurement across geographical and linguistic boundaries are less easily dealt with. Neuroimaging is particularly problematic because quantitative magnetic resonance imaging data are exquisitely sensitive to variations in imaging hard- and software and takes time to process. Genetic assays are largely consistent thanks to the years of work of the Psychiatric Genomics Consortium in aggregating data-sets ${ }^{11,12}$ but, like neuroimaging facilities, largely concentrated in European and North American research centres.

\section{Data sharing}

The data sharing required to build large and representative databases for such studies opens up technical issues, such as dealing with different scanners, imaging sequences and data processing at each site; legal and ethical issues about sharing data and the potential risks of identification; and, data management issues because of the size of the data files. ${ }^{22}$ Whole-genome genotyping, when linked to other information or data-sets, is a potential threat to anonymity and skull and cortex renderings from magnetic resonance imaging can also potentially identify individuals. The ENIGMA consortium has dealt with these issues by getting each site to analyse their own imaging and genetic data using common protocols, pooling the results by meta-analysis, and has thereby produced cutting edge insights into the impact of genetic variation on imaging indices and authoritative estimates on the differences between various psychiatric disorders and control samples. ${ }^{23}$ However, aggregating data reduces the richness of individual-level data-sets and can limit utility by obstructing the comparison of each participant to the predictive models.

\section{Informed consent (for research)}

The issue as to how much should be explained to people about the risks of various medical procedures is a live and unresolved one. Particular issues arise in the context of 'psychosis', if accompanied by a lack of awareness of or being out of touch with reality, with respect to the ability and/or competency to give informed consent. Consent and other ethical issues need also to be considered in particular populations, including patients with varying levels of comorbidity, intellectual disabilities and different understandings of mental illness and the meaning of genetic testing. Competency is, however, an abstract concept without an agreed definition or standardised assessment. Seeking consent from proxies, parents and advocates brings autonomy and confidentiality limitations, but is a potential solution in particular situations. Consent to data sharing can arguably never be fully informed regarding all current and potential future uses of data - giving rise to varying conceptions as to what extent individual autonomy is respected or not by broad research consent. Similar issues arise when analysing routine clinical health systems treatment and outcome data. Many countries are now adopting the acceptable compromise that fully anonymised data can be fairly safely exploited if one presumes consent unless people specifically 'opt out' of research using routinely collected data-sets. Indeed, we are currently engaged in participatory research with community stakeholders to study attitudes to routine sharing of health data of different types (see: https://mhdss.ac.uk). Additional safeguarding measures could include mandatory training for users of routine and linked data-sets (equivalent to Good Clinical Practice for clinical trials) and consultation with a stakeholder advisory group for each application of existing data to a new clinical or research question. However, such measures are not yet established.

\section{Feasibility in clinical practice}

Currently, mental health professionals in general adult psychiatry do not routinely use standardised assessments, because of concerns about their accuracy, utility and the time involved. It is, however, desirable that the data we use in clinical practice and in research is better harmonised. Routine collection of risk, treatment and outcome data in clinical practice could be used to inform patients, carers and health professionals about factors that could improve prevention and treatment for mental disorders. Although clinicians could probably be persuaded to adopt brief measurements if they were shown to improve patient care and outcomes, this would represent a substantial culture change. Further difficulties would arise if biological assays were to be included, given that genetic and imaging samples take time to process and communicate the results. The very sensitivity of neuroimaging to mental state means that it may both be among the strongest predictors but least reliable measures if strict protocols are not followed, which is not always possible in a busy clinical imaging environment, where 
planned sessions can be interrupted by the necessity of dealing with medical emergencies. Not everyone is prepared to have, or able to lie still for, a brain scan. Novel technologies such as portable neuroimaging devices may offer solutions here. In the meantime, PSYSCAN and PRONIA researchers are allowing for these issues of limited availability and technical complexity by building algorithms with and without imaging. Neuroimaging may prove to be unnecessary or prohibitively expensive; but patients may prefer imaging to cognitive tests that they find more demanding ${ }^{24}$ and symptom and cognitive assessments also share substantial variability. ${ }^{25}$ Multiple and sequential testing may be both optimally predictive and necessary for reliable clinical prediction, ${ }^{6}$ but raise logistical and financial issues. Blood tests for diagnosis and treatment prediction are therefore generally preferable - not least in low- and middle-income countries for example - but thus far none are available.

\section{Communication of risks and benefits}

One major issue that has hardly been addressed at all in research studies to date is how to develop and return results that are meaningful to individuals. After all, to render this technology suitable for clinical use, there is a need to be able to make individual predictions. Whereas a diagnostic test generates a binary outcome (disease present or absent), prediction tools return probabilistic outcomes (from $0 \%$ to $100 \%$ risk) that may have different implications for interventions in particular people at various risk levels. Further, these need to be available within a suitable time frame to be able to influence clinical management. In addition, they should preferably be linked to one or more interventions that are known to reduce the risk - even if that is, for example, brief interventions seeking to reduce cannabis consumption and thereby the risk of schizophrenia. Machine learning approaches, as currently employed, are largely unsuited to delivering individual results within short time frames. What is needed for clinical practice is something more akin to a normal reference range for an individuals' results to be compared with - similar to ' $N$-of-1' statistical testing ${ }^{26}$ and/or incorporated into a multivariate predictive model.

\section{Diagnosis}

One rarely remarked upon limitation of the 'psychosis' prediction studies done to date is that most include a range of psychotic disorders as part of the group of about one-third or so considered to have become psychotic. $^{7}$ But, a notable minority of these have brief or transient psychoses that do not typically need ongoing treatment. And others have for example delusional disorders that are often treated, with some clinical trial justification, differently from schizophrenia. There is also the consideration that about one-third of those with an at-risk mental state will develop other non-psychotic disorders. Similarly, depression can be transient and often spontaneously resolves within a year or so, but also has a tendency to recur. Predictive testing studies therefore need to consider various diagnostic and therapeutic outcomes, including when no treatment is desired or required.

\section{Early detection 'labelling' and other potential adverse effects}

The stigma of mental illness seems to be slowly reducing in some countries but remains a live concern. The contentious and often shameful history of psychiatry lives long in folk memory. Predictive testing, especially in general population screening rather than those at risk presenting to services, may smack to many of mind and behavioural control; and including genetics all too readily stokes memories of eugenics and fans fears of genetic discrimination. A central concern about early detection and possible intervention is that 'labelling' someone as at high risk may alter how they, their parents, teachers and others in authority may act towards them, and conceivably treat them in such a way as to cause stress or promote behaviours that may actually increase risk - and thereby even become a self-fulfilling prophecy. Equally, however, communication of risk could alter behaviour for the better, for example by improving diet or increasing physical activity, such as has been observed in some studies in those at high polygenic risk of heart disease.

Regardless, false positives and negatives are inevitable in any screening/early diagnostic programme, and the risks of these need to be balanced against the benefits for true positives and negatives. The risks of false-positive (and early correct) diagnoses include: potential consequences for employment and obtaining insurance, adversely having an impact on relationships, other and selfimposed restrictions and wider prejudice and discrimination. The risks of false-negative (and delayed correct) diagnoses include: exacerbating the condition through continued illicit drug use, stress and delayed treatment. Some of these risks may be ameliorated by communicating levels of risk rather than dichotomies (ideally accompanied by more widespread improvements in the statistical and scientific literacy of the general population). Of course, true positive results may benefit people through prompt treatment and likely illness outcome improvements, whereas people with true negatives might be reassured in the face of symptoms and/or a family history. But these are theoretical, somewhat speculative observations. We simply do not know if and how individuals may be affected because, as far we are aware, they have yet to be asked and the studies have yet to be done.

\section{Early intervention}

Above all, early detection research needs to be linked to therapeutic initiatives, and demonstrate advantages for patients, delivering outcomes that are endorsed by, and meaningful to, those affected. These should ideally be demonstrated through clinical trials (but such studies would be massive, very expensive undertakings). The availability of different treatments and knowledge of their effectiveness is likely to influence people's attitudes to early diagnostic testing and treatment. Indeed, desirable levels of the likely benefits and risks of various interventions might well alter acceptable rates of false-positive and false-negative diagnoses in different clinical scenarios. The AUC figures quoted above are balanced for sensitivity and specificity because we have little information about which treatments would be acceptable in different situations. Qualitative studies and large population-based questionnaire surveys are therefore required in various at-risk populations to see at what level of risk of depression or psychosis they might be prepared to take psychotherapeutic, behavioural or pharmacological interventions with known levels of beneficial and adverse effects. Further, demonstrating benefits to individuals taking part is an important influence on participation in screening.

In the rest of medicine, the uptake rate of cervical, breast and bowel cancer screening is encouraging, but that is for potentially fatal diseases for which there are increasingly effective treatments. Similarly, the routine use of cardiovascular disease risk reduction treatment thresholds has been shown to save lives. As regards neuropsychiatric disorders, Huntingtons chorea screening is much less successful, but then there is of course no effective treatment. For depression and psychosis, we do at least have good evidence that a range of interventions are more or less equally effective at improving outcomes in individuals with established illness. ${ }^{27}$ Further, a systematic review of ten randomised clinical trials has found that early intervention in psychosis reduces treatment discontinuation, psychiatric hospital admissions and 
symptom severity, and increases work involvement, over 6-24 months. ${ }^{28}$ Given, however, that psychotherapeutic approaches are generally preferred by patients, ${ }^{29}$ non-pharmacological treatments may be much more acceptable to those at high risk.

\section{Attitudes to research and preventative practice}

What do we know already about people's attitudes to such studies and their possible practical applications? A bit, but not much. During the Edinburgh High Risk Study of schizophrenia, ${ }^{9,14}$ up until 2010, and as part of the ongoing Bipolar Family Study, ${ }^{5}$ we held regular 'data parties' to feedback the results to the participants. The vast majority of those who were there and responded to surveys were supportive of the search for predictive diagnostic tests (unpublished data). They were of course, however, selected on the basis they were aged 18-25 at study outset, had at least two family members with schizophrenia, or mood disorders and were participating in such research.

Using a social media (Facebook) based survey we collected information in 2014 on general public opinions about pre-symptomatic testing and treatment for schizophrenia. The final questionnaire was live for a 2-week period during which we received 385 responses $(73 \%$ female) from young people predominantly in their twenties. The majority of respondents (67\%) indicated that they would consider a pre-symptomatic brain scan if they were at an increased risk of developing schizophrenia, $13 \%$ indicated that would have a brain scan if they were at even the $1 \%$ population risk, whereas $19 \%$ said that they would never have a brain scan.

Commonly voiced concerns included a potential waste of National Health Service resources and time, that it could be a traumatic event and that population risk was not enough to warrant screening. Most respondents (73\%) stated that they would be prepared to start pre-symptomatic treatment, but the threshold at which they would take treatment varied widely, with more or less equal numbers of people considering treatment across $0-100 \%$ decile risk levels. This showed no apparent relationship with knowledge about schizophrenia. Issues raised included insufficient evidence of the efficacy of early diagnosis and treatment in reducing the severity of the illness and potentially unnecessary exposure to medication. At around 50\% risk, most felt that the potential treatment benefits - of keeping jobs, relationships and future plans outweighed potential adverse effects (unpublished data).

From 2011 to 2015 we conducted an imaging and genetic study of a large pedigree multiply affected with a structural genetic variant of high penetrance and autosomal dominant like inheritance that increases the risk of major psychiatric disorders including depression, bipolar disorder and schizophrenia to more than $50 \%{ }^{30}$ We found ourselves in the somewhat contrary position, as dictated by ethics committees, of having to discuss any neuroimaging abnormalities with participants even if these were of no clinical relevance. On the other hand, we were simultaneously obliged to not reveal their translocation status, despite its obvious clinical implications, unless people specifically requested that we did. We found that the vast majority of the younger generations of the family did not wish to know whether or not they had the structural variant. This could reflect particular concerns about structural variants (which are relatively rare but of big effect, perhaps akin to Huntingtons chorea) and/or specific issues that have an impact on this unique family.

Finally, this year, as part of a meeting about longitudinal cohort studies concerned with mental health and illness in Scotland, we surveyed a similar number but a much wider age range of participants. The vast majority of attendees were either definitely (50\%) or on balance $(28 \%)$ prepared to have a research brain scan to help predict later mental illnesses such as schizophrenia or depression. And very similar proportions indicated that they themselves would definitely $(47 \%)$ or on balance $(26 \%)$ 'have a brain scan test of future mental illness, if it were safe and accurate'.

\section{Conclusions}

Early diagnosis and intervention in people at risk of developing schizophrenia or depression is technically feasible and has the potential to improve patient outcomes and even prevent disorder. Indeed, it is compatible with a public health, primary prevention approach. Risk factors such as social adversity and illicit drug use could potentially be mitigated, and have detectable epigenetic and other biological effects. ${ }^{31}$ However, identifying those at elevated risk is not straightforward and some people will inevitably be mis-classified as likely to become ill or not. There is therefore a need for further research to optimise accuracy and utility. Moreover, as with any such prevention strategy, there are important ethical considerations. Labelling someone as 'high risk' for a major mental illness could have a substantial impact on their lives. The case is even more complex when one considers the range of diagnoses and outcomes that are not in the category of 'major mental illness', and if there is no consistent and universally negative outcome to be avoided. Thus, definite overall individual and, probably also, societal benefit needs to accrue.

The limited information we have available to us suggests a tangible interest in early detection research and pre-symptomatic treatment for schizophrenia in a sizeable majority of almost 1000 people surveyed but found no clear risk level at which most people would accept treatment. Thus, there appears to be an appetite for such initiatives among both at-risk and general populations, but researchers need to systematically assess opinions and attitudes alongside technical advances. Key ethical considerations should be part and parcel of these scientific investigations - such as, what is it that people in these studies would want and are they typical of clinical populations such as those in early-psychosis services?

A coordinated programme of work is required to translate overall prediction profiles into individual risk scores, to find the optimal combination of variables to enhance predictive power, to test the acceptability of these approaches in relevant populations, and then go on to demonstrate their practicality, impact and costeffectiveness. This is a rather full and daunting agenda but brings with it the promise of improving the outcome of people with some of the most severe psychiatric disorders as well as providing objective diagnostic and therapeutic markers in psychiatry.

Stephen M. Lawrie, FRCPsych (iD, Head of Psychiatry, Division of Psychiatry and Patrick Wild Centre, University of Edinburgh, Scotland, UK; Sue Fletcher-Watson, PhD, Senior Lecturer, Division of Psychiatry and Patrick Wild Centre, University of Edinburgh, Scotland, UK; Heather C. Whalley, PhD, Senior Research Fellow, Division of Psychiatry, University of Edinburgh, Scotland, UK; Andrew M. McIntosh, FRCPsych, Professor of Biological Psychiatry, Division of Psychiatry and Centre for Cognitive Ageing and Cognitive Epidemiology, University of Edinburgh, Scotland, UK

Correspondence: Stephen M. Lawrie, Kennedy Tower, Royal Edinburgh Hospital, Edinburgh EH10 5HF, UK. Email: s.lawrie@ed.ac.uk

First received 15 Nov 2018, final revision 30 Jan 2019, accepted 31 Jan 2019

\section{Funding}

All four authors are supported by a MRC Pathfinder award: 'Leveraging routinely collected and linked research data to study the causes and consequences of common mental disorders' (MC) PC/17209).

\section{References}

1 Cooper D, Limet N, McClung I, Lawrie SM. Towards clinically useful neuroimaging in psychiatric practice. Br J Psychiatry 2013; 203: 242-4. 
2 Robinson L, Sprooten E, Lawrie SM. Brain imaging in psychosis and psychopathy-ethical considerations. Cortex 2011; 47: 1236-9.

3 Lawrie SM, Stanfield A, Johnstone EC, McIntosh AM. Predicting first episode psychosis in those at high risk for genetic or cognitive reasons. Epidemiol Psychiatr Sci 2012; 21: 323-8.

4 Bernardini F, Attademo L, Cleary SD, Luther C, Shim RS, Quartesan R, et al. Risk prediction models in psychiatry: toward a new frontier for the prevention of mental illnesses. J Clin Psychiatry 2017; 78: 572-83.

5 Whalley HC, Sussmann JE, Romaniuk L, Stewart T, Papmeyer M, Sprooten E, et al. Prediction of depression in individuals at high familial risk of mood disorders using functional magnetic resonance imaging. PLoS One 2013; 8: e57357.

6 Schmidt A, Cappucciati M, Radua J, Rutigliano G, Rocchetti M, Dell'Osso L, et al. Improving prognostic accuracy in subjects at clinical high risk for psychosis: systematic review of predictive models and meta-analytical sequential testing simulation. Schizophr Bull 2017; 43: 375-88.

7 Hunter SA, Lawrie SM. Imaging and genetic biomarkers predicting transition to psychosis. Curr Top Behav Neurosci 2018; 40: 353-88.

8 Cannon TD, Yu C, Addington J, Bearden CE, Cadenhead KS, Cornblatt BA, et al. An individualized risk calculator for research in prodromal psychosis. Am J Psychiatry 2016; 173: 980-8.

9 Zarogianni E, Storkey AJ, Johnstone EC, Owens DG, Lawrie SM. Improved individualized prediction of schizophrenia in subjects at familial high risk, based on neuroanatomical data, schizotypal and neurocognitive features. Schizophr Res 2017; 181: 6-12.

10 Lawrie SM. Clinical risk prediction in schizophrenia. Lancet Psychiatry 2014; 1 $406-8$

11 Schizophrenia Working Group of the Psychiatric Genomics Consortium. Biologica insights from 108 schizophrenia-associated genetic loci. Nature 2014; 511: 421-7.

12 Major Depressive Disorder Working Group of the Psychiatric Genomics Consortium. Genome-wide association analyses identify 44 risk variants and refine the genetic architecture of major depression. Nat Genet 2018; 50: 668-81.

13 Agerbo E, Sullivan PF, Vilhjálmsson BJ, Pedersen CB, Mors O, Børglum AD, et al. Polygenic risk score, parental socioeconomic status, family history of psychiatric disorders, and the risk for schizophrenia: a Danish population-based study and meta-analysis. JAMA Psychiatry 2015; 72: 635-41.

14 Neilson E, Bois C, Clarke TK, Hall L, Johnstone EC, Owens DGC, et al. Polygenic risk for schizophrenia, transition and cortical gyrification: a high-risk study. Psychol Med 2018; 48: 1532-9.

15 Lawrie SM, Olabi B, Hall J, McIntosh AM. Do we have any solid evidence of clinical utility about the pathophysiology of schizophrenia? World Psychiatry 2011 10: 19-31.

16 Alladi C G, Etain B, Bellivier F, Marie-Claire C. DNA methylation as a biomarker of treatment response variability in serious mental illnesses: a systematic review focused on bipolar disorder, schizophrenia, and major depressive disorder. Int J Mol Sci 2018; 19: E3026.

17 Fond G, d'Albis MA, Jamain S, Tamouza R, Arango C, Fleischhacker WW, et al. The promise of biological markers for treatment response in first-episode psychosis: a systematic review. Schizophr Bull 2015; 41: 559-73.
18 Marwood L, Wise $T$, Perkins AM, Cleare AJ. Meta-analyses of the neura mechanisms and predictors of response to psychotherapy in depression and anxiety. Neurosci Biobehav Rev 2018; 95: 61-72.

19 Lee Y, Ragguett RM, Mansur RB, Boutilier JJ, Rosenblat JD, Trevizol A, et al. Applications of machine learning algorithms to predict therapeutic outcomes in depression: a meta-analysis and systematic review. J Affect Disord 2018; 241: 519-32.

20 Drysdale AT, Grosenick L, Downar J, Dunlop K, Mansouri F, Meng Y, et al. Resting-state connectivity biomarkers define neurophysiological subtypes of depression. Nat Med 2017; 23: 28-38.

21 McGinn TG, Guyatt GH, Wyer PC, et al. Users' guides to the medical literature, XXII: how to use articles about clinical decision rules. JAMA 2000; 284: 79-84.

22 Dluhoš P, Schwarz D, Cahn W, van Haren N, Kahn R, Španiel F, et al. Multi-center machine learning in imaging psychiatry: a meta-model approach. Neuroimage 2017; 155: 10-24

23 Thompson PM, Stein JL, Medland SE, Hibar DP, Vasquez AA, Renteria ME. The ENIGMA Consortium: large-scale collaborative analyses of neuroimaging and genetic data. Brain Imaging Behav 2014; 8: 153-82.

24 Rose D, Papoulias C, MacCabe J, Walke J. Service users' and carers' views on research towards stratified medicine in psychiatry: a qualitative study. $B M C$ Res Notes 2015; 8: 489

25 Fisher AJ, Medaglia JD, Jeronimus BF. Lack of group-to-individual generalizability is a threat to human subjects research. Proc Natl Acad Sci U S A 2018; 115: E6106-15.

26 Johnstone M, Vasistha NA, Barbu MC, Dando O, Burr K, Christopher E, et al. Reversal of proliferation deficits caused by chromosome 16p13.11 microduplication through targeting NFKB signaling: an integrated study of patient-derived neuronal precursor cells, cerebral organoids and in vivo brain imaging. $\mathrm{Mol}$ Psychiatry 2019; 24: 294-311.

27 Fusar-Poli P, McGorry PD, Kane JM. Improving outcomes of first-episode psychosis: an overview. World Psychiatry 2017; 16: 251-65.

28 Correll CU, Galling B, Pawar A, Krivko A, Bonetto C, Ruggeri M, et al. Comparison of early intervention studies vs treatment as usual for early-phase psychosis: a systematic review, meta-analysis and meta-regression. JAMA Psychiatry 2018: 75: 555-65.

29 McHugh RK, Whitton SW, Peckham AD, Welge JA, Otto MW. Patient preference for psychological vs pharmacologic treatment of psychiatric disorders: a metaanalytic review. J Clin Psychiatry 2013; 74: 595-602.

30 Doyle OM, Bois C, Thomson P, Romaniuk L, Whitcher B, Williams SC, et al. The cortical thickness phenotype of individuals with DISC1 translocation resembles schizophrenia. J Clin Invest 2015; 125: 3714-22.

31 Yehuda R, Lehrner A. Intergenerational transmission of trauma effects: putative role of epigenetic mechanisms. World Psychiatry 2018; 17: 243-57. 